\title{
Does the mucosa activate or modulate the neural circuits controlling colonic motility?
}

\author{
Paul P. Bertrand* \\ Department of Physiology, School of Medical Sciences, University of New South Wales, Sydney, NSW, Australia \\ ${ }^{*}$ Correspondence: dr.p.bertrand@gmail.com
}

\section{A commentry on}

Localization of the sensory neurons and mechanoreceptors required for stretchevoked colonic migrating motor complexes in mouse colon

by Zagorodnyuk, V. P., and Spencer, N. J. (2011). Front. Physiol. 2:98. doi: 10.3389/ fphys.2011.00098

The neural control of the gastrointestinal (GI) tract is complex yet the tasks it has to accomplish are seemingly simple. One such task is to move the luminal contents down the intestine - from one area to another. Movement, or transit, of contents is critical for digestion and absorption but also for elimination of waste. While it is not yet clear how transit is controlled at the neuronal level there are many diseases which affect transit and this is a growing global health concern (Leung et al., 2011). In the large intestine, also called the large bowel or colon, a reduction in transit results in constipation. As a guideline, constipation is fewer than three bowel movements per week though bowel habits vary widely and Rome III criteria take into account many other factors (Drossman, 2006). There are many reasons why constipation can occur including drug use (e.g., opioids), GI tumors which obstruct the colon or pelvic floor defects which prevent emptying. Unfortunately, in many cases the reasons are unknown and fall under the umbrella of "functional bowel diseases," an example of which is slow transit constipation (STC; Leung et al., 2011). Current treatments with prokinetics (drugs which increase the propulsive motility of the colon) are promising, but not yet practical. For example, tegaserod, a 5- $\mathrm{HT}_{4}$ receptor agonist, facilitates colonic transit but was pulled from the general market following safety concerns - luckily new pharmacological treatments are on their way (Tack, 2011). The causes of slow colonic transit are unknown, but many subtle changes have been reported in the nerves, muscle, mucosa, and support cells throughout the colon. Thus, one impediment is that researchers have been spoilt for choice when investigating potential mechanisms underlying functional bowel diseases like STC.

Significant transit of contents in the human colon is thought to occur mainly during so-called "high amplitude propagating contractions," the correlate of which in animal models is the "colonic migrating motor complex" (CMMC). It is clear that the CMMC is run by the enteric nervous system (ENS), a network of neurons present within the wall of the small and large intestine which coordinates the functions of the GI tract (Furness, 2006). One class of neuron in the ENS is the sensory (afferent) neuron which, when connected to interneurons and motor neurons results in the movement of contents down the colon. What is not clear is how stimuli activate these sensory neurons - are they activated directly by mechanical forces, or perhaps indirectly by the release of other transmitter substances? One of the leading ideas is that stimulation of the mucosa and subsequent release of endocrine cell products initiates many mechanically evoked reflexes in the intestine. This process of sensory transduction in the intestine is itself a complex topic which has been reviewed previously in this journal (Bertrand, 2009).

The initiation of the CMMC in the mouse has recently been the focus of debate following several investigations designed to examine this motor pattern (e.g., Heredia et al., 2009; Keating and Spencer, 2010). One of the studies which is currently fanning the flames is a new paper by Zagorodnyuk and Spencer (2011) in this journal who have used a classical approach coupled with modern recordings techniques to unravel the initiation and propagation of colonic motor patterns. They hypothesized that if the mucosa were crucial for the CMMC, then a simple (but highly skilled) micro-dissection to remove the mucosa should not only reveal the role of 5-HT, but indeed a role for any substances from the mucosa. Using this technique, they have made some startling observations. Their study shows that 5-HT from the mucosa does not appear to be necessary for stretch-evoked CMMCs while a previous study showed similar data for spontaneously occurring CMMCs (Keating and Spencer, 2010). This area is of such interest that co-Editor-in-Chief Joel Bornstein has written a Commentary which cuts to the heart of the matter and asks the question, what role mucosal 5-HT has in the gut (Bornstein, 2012). While the role of 5-HT and the mucosa in CMMCs is being debated, it is worthwhile considering more closely three additional findings from Zagorodnyuk and Spencer which may shed some light on the issue.

In their study, Zagorodnyuk and Spencer (2011) observed a decrease in the frequency of the CMMC following the removal of the mucosa. This equates to an increase in the time between individual CMMCs and most likely means that the "gain" of the circuitry in the ENS has been reduced. Interestingly, increasing the overall excitability of the enteric circuitry is one role suggested for mucosal 5-HT (for review see Bertrand and Bertrand, 2010) based on evidence looking at mucosal application of 5-HT (e.g., Tuladhar et al., 1997) and more recently 5- $\mathrm{HT}_{4}$ receptor agonists (Hoffman et al., 2012). Second, the threshold for initiation of the CMMC was decreased, that is, it was easier to evoke a CMMC. At first glance, this seems to be the opposite of any prediction which has mucosal 5-HT enhancing the reflex. However, the mucosa and submucosa form a strong, mechanically stable component of the preparation. With the submucosa gone, the mechanical properties of the remaining longitudinal muscle, myenteric plexus and circular muscle may 
be very different. Thus, while these data show clearly that the CMMC can still be evoked, the exact threshold for initiation with or without the submucosa may not provide a useful comparison. Finally, the authors identified mechanical stimulation of myenteric ganglion as a possible contributor to the initiation of the reflex. During stretch, myenteric ganglia might undergo some uniform compression, however, mechanical stimulation of the mucosa might cause non-uniform or localized deformation of ganglia. Many studies have used compression or stroking of the mucosa as a stimulus, so the question for future studies is, does the mechanosensitivity of the ganglia account for the initiation of some, or even all, of these reflexes?

In conclusion, Zagorodnyuk and Spencer (2011) have found that the mucosa does not contribute to the initiation of the CMMC. Indeed, their data shows that the role of 5-HT in the colon is not to activate stretch reflexes but - and perhaps as importantly - to modulate these reflexes. These data may help to reduce the number of choices for researchers investigating chronic constipation and help focus on either the initiation or modulation of the motor reflexes responsible for colonic transit. Although the tasks which the GI tract accomplishes are simple on the surface, the nerve circuits underlying these reflexes are surprisingly complex. The results of the study by Zagorodnyuk and Spencer provide an excellent working model for the further dissection of the always interesting, and in some cases exasperating, neural control of the GI tract.

\section{REFERENCES}

Bertrand, P. P. (2009). The cornucopia of intestinal chemosensory transduction. Front. Neurosci. 3:48. doi: 10.3389/neuro.21.003.2009

Bertrand, P. P., and Bertrand, R. L. (2010). Serotonin release and uptake in the gastrointestinal tract. Auton. Neurosci. 153, 47-57.

Bornstein, J. C. (2012). Serotonin in the gut: what does it do? Front. Neurosci. 6:16. doi: 10.3389/ fnins.2012.00016

Drossman, D. A. (2006). The functional gastrointestinal disorders and the Rome III process. Gastroenterology 130, 1377-1390.

Furness, J. B. (2006). The Enteric Nervous System. Oxford, UK: Wiley-Blackwell.

Heredia, D. J., Dickson, E. J., Bayguinov, P. O., Hennig, G. W., and Smith, T. K. (2009). Localized release of serotonin (5-hydroxytryptamine) by a fecal pellet regulates migrating motor complexes in murine colon. Gastroenterology 136, 1328-1338.

Hoffman, J. M., Tyler, K., Maceachern, S. J., Balemba, O. B., Johnson, A. C., Brooks, E. M., Zhao, H., Swain, G. M., Moses, P. L., Galligan, J. J., Sharkey, K. A., Meerveld, B. G., and Mawe, G. M. (2012). Activation of colonic mucosal 5-HT 4 receptors accelerates propulsive motility and inhibits visceral hypersensitivity. Gastroenterology. doi: 10.1053/j. gastro.2011.12.041

Keating, D. J., and Spencer, N. J. (2010). Release of 5 -hydroxytryptamine from the mucosa is not required for the generation or propagation of colonic migrating motor complexes. Gastroenterology 138, 659-670.

Leung, L., Riutta, T., Kotecha, J., and Rosser, W. (2011). Chronic constipation: an evidence-based review. J. Am. Board Fam. Med. 24, 436-451.

Tack, J. (2011). Current and future therapies for chronic constipation. Best Pract. Res. Clin. Gastroenterol. 25, 151-158.

Tuladhar, B. R., Kaisar, M., and Naylor, R. J. (1997). Evidence for a 5-HT3 receptor involvement in the facilitation of peristalsis on mucosal application of 5-HT in the guinea pig isolated ileum. $\mathrm{Br} . \mathrm{J}$. Pharmacol. 122, 1174-1178.

Zagorodnyuk, V.P., and Spencer, N. J. (2011). Localization of the sensory neurons and mechanoreceptors required for stretch-evoked colonic migrating motor complexes in mouse colon. Front. Physiol. 2:98. doi: 10.3389/fphys.2011.00098

Received: 17 January 2012; accepted: 28 February 2012; published online: 26 March 2012.

Bertrand PP (2012) Does the mucosa activate or modulate the neural circuits controlling colonic motility? Front. Neurosci. 6:38. doi:10.3389/fnins.2012.00038

This article was submitted to Frontiers in Autonomic Neuroscience, a specialty of Frontiers in Neuroscience.

Copyright (C) 2012 Bertrand. This is an open-access article distributed under the terms of the Creative Commons Attribution Non Commercial License, which permits non-commercial use, distribution, and reproduction in other forums, provided the original authors and source are credited. 